\title{
SUMS OF POWERS OF DERIVATIVES
}

\author{
JAN MAŘÍK AND CLIFFORD E. WEIL
}

(Communicated by Andrew M. Bruckner)

\begin{abstract}
In a previous paper the authors proved several theorems concerning products of positive derivatives. Here they prove analogous results for sums of powers of positive derivatives.
\end{abstract}

\section{INTRODUCTION}

In [1] the authors proved that if the product of powers of several positive derivatives is approximately continuous and if the corresponding exponents are positive, then all of the factors must be approximately continuous. The main goal of this work is to prove some analogous results for sums of powers of derivatives. For example, it follows from Theorem 5.6 that if the sum of squares of several derivatives is bounded and approximately continuous, then all of these derivatives are approximately continuous.

The authors also investigate equations like $f^{2}+g^{2}=\varphi^{2}$, where $f, g$, and $\varphi$ are derivatives. We can construct nontrivial examples of such triples $f, g$, $\varphi$, if we choose a bounded derivative $\psi$, bounded approximately continuous functions $\alpha, \beta$, and set $f=\alpha \psi, g=\beta \psi, \varphi=\psi \sqrt{\alpha^{2}+\beta^{2}}$. Theorem 5.11 shows that this construction is "not too far" from the general case.

Some of the results of this paper have been stated without proof in $[2,3]$.

\section{NotATION}

The word "function" means a mapping to the real line $R$. The words "measure," "integrable", etc. refer to Lebesgue measure in $R$. The measure of a measurable set $S \subset R$ will be denoted by $|S|$. Symbols like $\int_{S} f, \int_{a}^{b} f$ will always denote the corresponding Lebesgue integrals.

The letter $m$ denotes a natural number and $R^{m}$ is $m$-dimensional Euclidean space. The coordinates of a point $a \in R^{m}$ will be denoted usually by $a_{1}, \ldots, a_{m}$. For $x, y \in R^{m}$ we set $x \cdot y=\sum_{i=1}^{m} x_{i} y_{i}$ and $\|x\|=\sqrt{x \cdot x}$. If $|\cdot|$ is any norm on $R^{m}$ and if $f_{1}, \ldots, f_{m}$ are functions on a set $S$, then

Received by the editors January 17, 1990.

1980 Mathematics Subject Classification (1985 Revision). Primary 26A24. 
$\left\{\left(f_{1}, \ldots, f_{m}\right) \mid\right.$ means, of course, the function $\left|\left(f_{1}(t), \ldots, f_{m}(t)\right)\right|(t \in S)$.

\section{Preliminary Results}

In this section we investigate some special norms on $R^{m}$ (see 3.4). The corresponding results will be used later in this paper. They are valid, in particular, for the norms

$$
\|x\|_{p}=\left(\sum_{i=1}^{m}\left|x_{i}\right|^{p}\right)^{1 / p} \quad(p>1)
$$

(hence $\|x\|=\|x\|_{2}$ ); actually, for the proofs of the main results (Theorems 5.5, 5.6, and 5.11) we will need no other norms. However, the reader might find it interesting that the results of $\S 4$ hold for more general norms.

3.1. Lemma. Let $V$ be a vector space over $R,|\cdot|$ a norm on $V$, and $x$, $y \in V$. Suppose that $|t x+(1-t) y|=1$ for each $t \in[0,1]$. Then

$$
|\alpha x+\beta y| \geqq \alpha+\beta \quad \text { for all } \alpha, \beta \in R \text {. }
$$

Proof. We may suppose that $\alpha+\beta>0$. We have

$$
|\alpha x+\beta y|=(\alpha+\beta)\left|\frac{\alpha}{\alpha+\beta} x+\frac{\beta}{\alpha+\beta} y\right|=\alpha+\beta \quad \text { if } \alpha, \beta \geqq 0 .
$$

If, e.g., $\alpha<0$, then $0<\beta=|\beta y| \leqq|\alpha x+\beta y|+|-\alpha x|=|\alpha x+\beta y|-\alpha$ so that $\alpha+\beta<|\alpha x+\beta y|$.

3.2. Proposition. Let $H$ be a Hilbert space over $R$ with the inner product denoted by $x \cdot y$. Let $|\cdot|$ be a norm on $H$ which generates the same topology as the inner product. (We do not assume that $|x|=\sqrt{x \cdot x}$.) Suppose that $x$, $y \in H$ and $|t x+(1-t) y|=1$ for each $t \in[0,1]$. Then there is $a b \in H$ such that $x \cdot b=y \cdot b=1$ and $z \cdot b \leqq|z|$ for all $z \in H$.

Proof. The set $V=\{\alpha x+\beta y ; \alpha, \beta \in R\}$ is clearly a vector space over $R$. If $\alpha x+\beta y=0$, then, by 3.1, $\alpha+\beta=0$. Hence we may define a linear functional $\Phi$ on $V$ setting $\Phi(\alpha x+\beta y)=\alpha+\beta$. According to 3.1 we have $|\Phi(v)| \leqq|v|$ for each $v \in V$. By the Hahn-Banach theorem $\Phi$ has a linear extension $\Phi^{*}$ to all of $H$ such that $\left|\Phi^{*}(z)\right| \leqq|z|$ for each $z \in H$. By assumption, $\Phi^{*}$ is continuous in the topology induced by the inner product. It follows from the Riesz Representation Theorem that there is a $b \in H$ such that $\Phi^{*}(z)=z \cdot b$ for all $z \in H$. Then $x \cdot b=\Phi(x)=1=\Phi(y)=y \cdot b$ and $z \cdot b=\Phi^{*}(z) \leqq|z|$ for all $z \in H$.

3.3. Proposition. Let $|\cdot|$ be a norm on $R^{m}$ and let $f_{1}, \ldots, f_{m}$ be functions integrable on $R$. Set $f=\left(f_{1}, \ldots, f_{m}\right), A=\left(\int_{R} f_{1}, \ldots, \int_{R} f_{m}\right)$. Then $|A| \leqq$ $\int_{R}|f|$.

Proof. It may be assumed that $|A|>0$. By 3.2 with $x=y=A /|A|$ there is a $b \in R^{m}$ such that $b \cdot A=|A|$ and $b \cdot z \leqq|z|$ for all $z \in R^{m}$. In particular, $b \cdot f(t) \leqq|f(t)|$ for each $t \in R$. Thus $|A|=\int_{R} b \cdot f \leqq \int_{R}|f|$. 
3.4. Convention, notation. For the remainder of this paper, $|\cdot|$ is a norm on $R^{m}$ and $Z=\left\{z \in R^{m} ;|z|=1\right\}$. It is well known that there are positive numbers $k, K$ such that

$$
k|x| \leqq\|x\| \leqq K|x| \text { for each } x \in R^{m} .
$$

We shall assume that the following conditions are satisfied:

$\left(C_{1}\right)$ For each $x=\left(x_{1}, \ldots, x_{m}\right)$ we have $|x|=\left|\left(\left|x_{1}\right|, \ldots,\left|x_{m}\right|\right)\right|$.

$\left(\mathrm{C}_{2}\right)$ For each $x \in Z$ there is a unique $b \in R^{m}$ such that $x \cdot b=1$ and that $y \in Z, y \neq x$ implies $y \cdot b<1$.

We define a function $\theta$ on $Z$ setting $\theta(x)=b$, where $b$ is determined by $\left(\mathrm{C}_{2}\right)$.

Remark. We will prove in 5.3 that the norms $\|x\|_{p} \quad(p>1)$ satisfy conditions $\left(\mathrm{C}_{1}\right)$ and $\left(\mathrm{C}_{2}\right)$ with $b=\left(b_{1}, \ldots, b_{m}\right)$, where $b_{i}=\left|x_{i}\right|^{p-1} \operatorname{sgn} x_{i}$.

3.5. Lemma. Let $x \in Z, b \in R^{m}$. Suppose that $x \cdot b=1$ and $y \cdot b \leqq 1$ for each $y \in Z$. Then $b=\theta(x)$.

Proof. Set $c=\frac{1}{2}(b+\theta(x))$. Then $x \cdot c=1$ and $y \cdot c<1$ for each $y \in Z \backslash\{x\}$. By the uniqueness part of $\left(\mathrm{C}_{2}\right)$ we have $c=\theta(x)$ so that $b=\theta(x)$.

3.6. Lemma. Let $0 \leqq x_{i} \leqq y_{i}$ for $i=1, \ldots, m$. Then $|x| \leqq|y|$.

Proof. Set $a=\left(y_{1}, x_{2}, \ldots, x_{m}\right), b=\left(-y_{1}, x_{2}, \ldots, x_{m}\right)$. First we show that $|x| \leqq|a|$. This is obvious, if $y_{1}=0$, since in this case $x=a$. If $y_{1}>0$, we set $\alpha=\left(y_{1}+x_{1}\right) / 2 y_{1}, \beta=\left(y_{1}-x_{1}\right) / 2 y_{1}$ and we have $\alpha+\beta=1, x=\alpha a+\beta b$. By $\left(\mathrm{C}_{1}\right)$ we have $|a|=|b|$. Thus $|x| \leqq \alpha|a|+\beta|b|=|a|$. We can prove similarly that $|a| \leqq\left|\left(y_{1}, y_{2}, x_{3}, \ldots, x_{m}\right)\right|$, etc.

3.7. Lemma. Let $0 \leqq x_{i} \leqq y_{i}$ for $i=1, \ldots, m$ and let $|x|=|y|$. Then $x=y$.

Proof. One may assume that $x, y \in Z$. Choose a $t \in[0,1]$ and set $a=$ $t x+(1-t) y$. It follows from 3.6 that $|x| \leqq|a| \leqq|y|$. Since $|x|=|y|=1$, we also have $|a|=1$. By 3.2 there is a $b \in R^{m}$ such that $x \cdot b=y \cdot b=1$ and $z \cdot b \leqq 1$ for each $z \in Z$. By 3.5 we have $b=\theta(x)$ and it follows from $\left(\mathrm{C}_{2}\right)$ that $y=x$.

\subsection{Lemma. The mapping $\theta$ is continuous.}

Proof. Let $k$ be as in (1) and $x \in Z$. Set $b=\theta(x), y=b /|b|$. Then $y \in Z$ and consequently $b \cdot b /|b|=y \cdot b \leqq 1$. Hence $\|b\|^{2}=b \cdot b \leqq|b| \leqq k^{-1}\|b\|$ so that $\|b\| \leqq k^{-1}$. We see that $\theta$ is bounded.

Let $x, x_{1}, x_{2}, \ldots \in Z, x_{n} \rightarrow x$. Suppose that the relation $\theta\left(x_{n}\right) \rightarrow \theta(x)$ does not hold. Then by the boundedness of $\theta$ there is a subsequence $\left\langle z_{n}\right\rangle$ of $\left\langle x_{n}\right\rangle$ and a $b \in R^{m}$ such that $\theta\left(z_{n}\right) \rightarrow b \neq \theta(x)$. Let $y \in Z$. By $\left(\mathrm{C}_{2}\right)$ we have $z_{n} \cdot \theta\left(z_{n}\right)=1$ and $y \cdot \theta\left(z_{n}\right) \leqq 1$ for each $n$. Hence $x \cdot b=1$ and $y \cdot b \leqq 1$. By $3.5, b=\theta(x)$ which is a contradiction. 
3.9. Lemma. Let $\varepsilon \in(0,1)$. Then there is a $P \in(0,1)$ such that $y \cdot \theta(x) \leqq P$, whenever $x, y \in Z$ and $\|y-x\| \geqq \varepsilon$.

(This follows easily from 3.8.)

3.10. Notation. For each function $g$ defined on $(0,1)$ we set

$$
\lambda(g)=\liminf g(t), \quad \text { and } \Lambda(g)=\limsup g(t) \quad(t \rightarrow 0+) \text {. }
$$

If $f=\left(f_{1}, \ldots, f_{m}\right)$ is a mapping of $(0,1)$ to $R^{m}$, we define $\lambda(f)=$ $\left(\lambda\left(f_{1}\right), \ldots, \lambda\left(f_{m}\right)\right), \Lambda(f)=\left(\Lambda\left(f_{1}\right), \ldots, \Lambda\left(f_{m}\right)\right)$.

3.11. Lemma. Let $f_{1}, \ldots, f_{m}$ be nonnegative functions defined on $(0,1)$ and let $f=\left(f_{1}, \ldots, f_{m}\right)$. Suppose that $\lambda(|f|)<\infty$. Then $|\lambda(f)| \leq \lambda(|f|)$.

Proof. There are $t_{1}, t_{2}, \ldots \in(0,1)$ such that $t_{n} \rightarrow 0$ and that $\left|f\left(t_{n}\right)\right| \rightarrow$ $\lambda(|f|)$. Let $K$ be as in (1). Then

$$
f_{i}\left(t_{n}\right) \leqq K\left|f\left(t_{n}\right)\right| \quad \text { for all } i, n \text {. }
$$

Since $\lambda(|f|)<\infty$, the sequences $\left\langle f_{i}\left(t_{n}\right)\right\rangle \quad(i=1, \ldots, m)$ are bounded. Thus there is a subsequence $\left\langle v_{n}\right\rangle$ of $\left\langle t_{n}\right\rangle$ and a $b=\left(b_{1}, \ldots, b_{m}\right) \in R^{m}$ such that $f\left(v_{n}\right) \rightarrow b$. Clearly $\lambda\left(f_{i}\right) \leqq b_{i}$ for each $i$. By 3.6 we have $|\lambda(f)| \leqq|b|=$ $\lim \left|f\left(v_{n}\right)\right|=\lambda(|f|)$.

3.12. Lemma. Let $f_{i}$ and $f$ be as in 3.11. Suppose that $\Lambda(|f|)<\infty$. Let $b_{1}=\Lambda\left(f_{1}\right), b_{2}=\lambda\left(f_{2}\right), \ldots, b_{m}=\lambda\left(f_{m}\right), b=\left(b_{1}, \ldots, b_{m}\right)$. Then $|b| \leqq$ $\Lambda(|f|)$.

Proof. There are $t_{1}, t_{2}, \ldots \in(0,1)$ such that $t_{n} \rightarrow 0$ and $f_{1}\left(t_{n}\right) \rightarrow b_{1}$. Since $\Lambda(|f|)<\infty$, by (2) the sequences $\left\langle f_{i}\left(t_{n}\right)\right\rangle \quad(i=1, \ldots, m)$ are bounded. Thus there is a subsequence $\left\langle v_{n}\right\rangle$ of $\left\langle t_{n}\right\rangle$ and a $B=\left(B_{1}, \ldots, B_{m}\right) \in R^{m}$ such that $f\left(v_{n}\right) \rightarrow B$. Clearly $b_{i} \leqq B_{i} \leqq \Lambda\left(f_{i}\right)$ for each $i$. By 3.6 we have $|b| \leqq|B|=\lim \left|f\left(v_{n}\right)\right| \leqq \Lambda(|f|)$.

\section{NORMS AND CONVEXITY}

4.1. Notation. Let $\mathscr{I}$ be the system of all functions integrable on $[0,1]$. Let

$$
\begin{aligned}
& \mathscr{D}_{*}=\left\{f \in \mathscr{I} ; f(0) \leqq \liminf t^{-1} \int_{0}^{t} f(t \rightarrow 0+)\right\}, \\
& \mathscr{D}^{*}=\left\{f \in \mathscr{I} ; f(0) \geqq \limsup t^{-1} \int_{0}^{t} f(t \rightarrow 0+)\right\},
\end{aligned}
$$

and $\mathscr{D}=\mathscr{D}_{*} \cap \mathscr{D}^{*}$. Let $\mathscr{A}$ be the system of all functions that are measurable on $[0,1]$ and approximately continuous from the right at 0 and let

$$
\mathscr{L}=\left\{f \in \mathscr{I} ; t^{-1} \int_{0}^{t}|f-f(0)| \rightarrow 0(t \rightarrow 0+)\right\} .
$$

(Thus $\mathscr{L}$ is the system of all functions $f$ measurable on $[0,1]$ for which $|f-f(0)| \in \mathscr{D}$.) For any system $\mathscr{T}$ of functions let $\mathscr{T}^{+}[b \mathscr{T}]$ be the system of all nonnegative [bounded] elements of $\mathscr{T}$. 
Remark. Each of the systems $\mathscr{I}, \mathscr{D}, \mathscr{A}, \mathscr{L}$ is a vector space. It is easy to see that $b \mathscr{A} \subset \mathscr{L} \subset \mathscr{D} \cap \mathscr{A}$.

4.2. Lemma. Let $f, g \in \mathscr{A},|g| \leqq f \in \mathscr{D}$. Then $g \in \mathscr{L}$.

Proof. We may suppose that $g \geqq 0$. Set $c=f(0)+1, f_{0}=f \wedge c, g_{0}=g \wedge c$, $f_{1}=f-f_{0}, g_{1}=g-g_{0}$. Since $f_{0} \in b \mathscr{A}$, we have $f_{0} \in \mathscr{L}$; similarly $g_{0} \in \mathscr{L}$. From the relations $g_{1}=(g-c) \vee 0 \leqq(f-c) \vee 0=f_{1}$ and $t^{-1} \int_{0}^{t} f_{1}=$ $t^{-1} \int_{0}^{t} f-t^{-1} \int_{0}^{t} f_{0} \rightarrow f(0)-f_{0}(0)=0$ we get $t^{-1} \int_{0}^{t} g_{1} \rightarrow 0(t \rightarrow 0+)$ so that $g_{1} \in \mathscr{L}$. Hence $g \in \mathscr{L}$.

4.3. Lemma. Let $f \in \mathscr{D}$. Let $J$ be an open interval containing $f([0,1])$, let $\gamma$ be a convex function on $J$ and let $\gamma \circ f \in \mathscr{I}$. Then $\gamma \circ f \in \mathscr{D}_{*}$.

Proof. There is a linear function $\mu$ such that $\mu(f(0))=\gamma(f(0))$ and that $\mu \leqq \gamma$ on $J$. Obviously $\mu \circ f \in \mathscr{D}$ so that $\gamma(f(0))=\mu(f(0))=\lim t^{-1} \int_{0}^{t} \mu \circ f \leqq$ $\liminf t^{-1} \int_{0}^{t} \gamma \circ f(t \rightarrow 0+)$.

4.4. Lemma. Let $f$ and $J$ be as in 4.3. Let $\gamma$ be a strictly convex function on $J$ and let $\gamma \circ f \in \mathscr{D}^{*}$. Then $f, \gamma \circ f \in \mathscr{L}$.

Proof. Set $c=f(0)$. There is a linear function $\mu$ such that $\mu(c)=\gamma(c)$ and that $\mu<\gamma$ on $J \backslash\{c\}$. Set $\psi=\gamma-\mu$. By assumption and 4.3 we have $\gamma \circ f \in \mathscr{D}$ so that $\psi \circ f \in \mathscr{D}$. Since $\psi \circ f \geqq 0$ and $\psi(f(0))=0$, we have $\psi \circ f \in \mathscr{L}$.

Choose an $\varepsilon \in(0, \infty)$ such that $c \pm \varepsilon \in J$ and set $\eta=\min \left(\psi^{\prime+}(c+\varepsilon)\right.$, $\left.\left|\psi^{\prime-}(c-\varepsilon)\right|\right), \psi_{0}(y)=\eta(|y-c|-\varepsilon) \quad(y \in J)$. If $|y-c|<\varepsilon$, then $\psi_{0}(y)<0$. If $y \in J \cap[c+\varepsilon, \infty)$, then $\psi(y) \geqq \psi(c+\varepsilon)+\psi^{\prime+}(c+\varepsilon)(y-c-\varepsilon)>\psi_{0}(y)$; it can be proved in a similar way that $\psi>\psi_{0}$ on $J \cap(-\infty, c-\varepsilon]$. Hence $\psi>\psi_{0}$ on $J$. It follows that $|y-c|<\eta^{-1} \psi(y)+\varepsilon$ for each $y \in J$ and that $\int_{0}^{t}|f-c| \leqq \eta^{-1} \int_{0}^{t} \psi \circ f+\varepsilon t$ for each $t \in[0,1]$. This together with the relation $\psi \circ f \in \mathscr{D}$ proves that $f \in \mathscr{L}$. Hence $\gamma \circ f=\psi \circ f+\mu \circ f \in \mathscr{L}$ as well.

4.5. Lemma. Let $f_{1}, \ldots, f_{m} \in \mathscr{D}_{*}^{+}, f=\left(f_{1}, \ldots, f_{m}\right)$ and let $|f| \in \mathscr{D}^{*}$. Then $f_{1}, \ldots, f_{m},|f| \in \mathscr{D}$.

Proof. For $t \in(0,1)$ and $i=1, \ldots, m$ set $F_{i}(t)=t^{-1} \int_{0}^{t} f_{i}$; further set $F=\left(F_{1}, \ldots, F_{m}\right)$. Proposition 3.3 implies that

$$
|F(t)| \leqq t^{-1} \int_{0}^{t}|f| \text { for each } t \in(0,1) .
$$

Since $f_{i}(0) \leqq \lambda\left(F_{i}\right)$, we have by 3.6 and $3.11,|f(0)| \leqq|\lambda(F)| \leqq \lambda(|F|)$ which together with (3) shows that $|f| \in \mathscr{D}_{*}$.

Now define $b_{1}=\Lambda\left(F_{1}\right), b_{2}=\lambda\left(F_{2}\right), \ldots, b_{m}=\lambda\left(F_{m}\right), b=\left(b_{1}, \ldots, b_{m}\right)$. It follows from 3.6 and 3.12 that $|f(0)| \leqq|b| \leqq \Lambda(|F|)$. Since $|f| \in \mathscr{D}^{*}$, we have by $(3), \Lambda(|F|) \leqq|f(0)|$. Hence $|f(0)|=|b|$. By 3.7 we have $f_{1}(0)=b_{1}=\Lambda\left(F_{1}\right)$ whence $f_{1} \in \mathscr{D}^{*}$. Similarly $f_{2}, \ldots, f_{m} \in \mathscr{D}^{*}$.

4.6. Proposition. Let $f_{1}, \ldots, f_{m} \in \mathscr{D}$. Let $f=\left(f_{1}, \ldots, f_{m}\right), \varphi=|f|$, $c=\liminf \operatorname{ap} \varphi(t) \quad(t \rightarrow 0+)$. Suppose that $\varphi \in \mathscr{D}$ and that $c>0$. Then $\varphi(0)>0$ and there are $\alpha_{1}, \ldots, \alpha_{m} \in \mathscr{A}$ such that $f_{i}=\alpha_{i} \varphi \quad(i=1, \ldots, m)$. 
Proof. It follows easily from the relation $\varphi \in \mathscr{D}^{*}$ that $\varphi(0) \geqq c$. Define $J=[0,1], \alpha_{i}(t)=f_{i}(t) / \varphi(t)$, if $\varphi(t)>0$, and $\alpha_{i}(t)=\alpha_{i}(0)$ elsewhere on $J$. Then $f_{i}=\alpha_{i} \varphi$ on $J$ for $i=1, \ldots, m$. Let $\alpha=\left(\alpha_{1}, \ldots, \alpha_{m}\right)$ and $\int_{0}^{t} f=$ $\left(\int_{0}^{t} f_{1}, \ldots, \int_{0}^{t} f_{m}\right)$ for each $t \in J$. Let $\varepsilon \in(0,1)$. Since $f_{1}, \ldots, f_{m} \in \mathscr{D}$, we have $\int_{0}^{t} f /\left|\int_{0}^{t} f\right| \rightarrow f(0) /|f(0)|=\alpha(0) \quad(t \rightarrow 0+)$. Thus there is a $\delta \in(0,1)$ such that

$$
\left\|\int_{0}^{t} f /\left|\int_{0}^{t} f\right|-\alpha(0)\right\|<\varepsilon \text { for each } t \in(0, \delta) .
$$

Set $E=\{t \in J ;\|\alpha(t)-\alpha(0)\|>2 \varepsilon\}$ and choose a $P \in(0,1)$ according to 3.9. Let $x \in(0, \delta), A=\int_{0}^{x} f, A_{1}=A /|A|, B=\theta\left(A_{1}\right)$. Then $A \cdot B=|A|$. If $t \in E \cap(0, x)$, then from (4) $\left\|\alpha(t)-A_{1}\right\| \geqq\|\alpha(t)-\alpha(0)\|-\left\|A_{1}-\alpha(0)\right\|>$ $2 \varepsilon-\varepsilon=\varepsilon$. Consequently $\alpha(t) \cdot B \leqq P, f(t) \cdot B \leqq P \varphi(t)$. Clearly, $f \cdot B \leqq \varphi$ on $J$.

Choose an $\eta \in(0, c)$ and set $T=\{t \in J ; \varphi(t)>\eta\}, S=T \cap E \cap(0, x)$, $S^{\prime}=(0, x) \backslash S$. Since $\varphi \in \mathscr{D}$, there is a $Q \in(0, \infty)$ such that $\int_{0}^{t} \varphi \leqq Q t$ for each $t \in J$. We have

$$
\left|\int_{0}^{x} f\right|=A \cdot B=\int_{0}^{x} f \cdot B \leqq P \int_{S} \varphi+\int_{S^{\prime}} \varphi=\int_{0}^{x} \varphi-(1-P) \int_{S} \varphi .
$$

Obviously

$$
\int_{S} \varphi \geqq|S| \eta \geqq(|S| \eta / Q x) \int_{0}^{x} \varphi
$$

so that

$$
\left|\int_{0}^{x} f\right| \leqq(1-(1-P)|S| \eta / Q x) \int_{0}^{x} \varphi .
$$

Combining this inequality with the relation $\left|\int_{0}^{x} f\right| / \int_{0}^{x} \varphi \rightarrow|f(0)| / \varphi(0)=1$ we obtain $|T \cap E \cap(0, x)| / x \rightarrow 0 \quad(x \rightarrow 0+)$. Since $E \subset(E \cap T) \cup(J \backslash T)$ and $|T \cap(0, x)| / x \rightarrow 1$, we have also $|E \cap(0, x)| / x \rightarrow 0 \quad(x \rightarrow 0+)$. This proves that $\alpha_{1}, \ldots, \alpha_{m} \in \mathscr{A}$.

4.7. Convention. In 4.8-4.11 we shall use the following notation and assumptions: $\mathscr{M}=\{1, \ldots, m\} ; \mathscr{N} \subset \mathscr{M} ; f_{1}, \ldots, f_{m} \in \mathscr{D} ;$ for each $i \in \mathscr{N}$ a strictly convex function $\gamma_{i}$ on $R$ is given such that $\gamma_{i}(t)>0$, if $t \neq 0 ; g_{i}=\gamma_{i} \circ f_{i}$ for $i \in \mathscr{N}, g_{i}=f_{i}$ for $i \in \mathscr{M} \backslash \mathscr{N} ; \varphi=\left|\left(g_{1}, \ldots, g_{m}\right)\right| \in \mathscr{D}^{*}$.

4.8. Proposition. We have

$$
g_{1}, \ldots, g_{m},\left|g_{1}\right|, \ldots,\left|g_{m}\right|, \varphi \in \mathscr{D}
$$

and

$$
f_{i}, g_{i} \in \mathscr{L} \text { for each } i \in \mathscr{N} .
$$

Proof. Since $\left|g_{i}\right| \leqq\left\|\left(g_{1}, \ldots, g_{m}\right)\right\|$, we get by (1)

$$
\left|g_{i}\right| \leqq K \varphi
$$


so that $g_{i} \in \mathscr{I}$. Let $h_{i}=\left|g_{i}\right|$. By 4.3 we have $h_{i} \in \mathscr{D}_{*} \quad(i \in \mathscr{M})$. It follows from $\left(\mathrm{C}_{1}\right)$ in 3.4 and from 4.5 that $\varphi=\left|\left(h_{1}, \ldots, h_{m}\right)\right|$ and that $h_{1}, \ldots, h_{m}$, $\varphi \in \mathscr{D}$. This proves (5); now 4.4 implies (6).

4.9. Proposition. If

$$
\varphi(0)=0
$$

or

$$
f_{i}(0) \neq 0 \quad \text { for some } i \in \mathscr{N} \text {, }
$$

then

$$
f_{1}, \ldots, f_{m}, \varphi \in \mathscr{L} \text {. }
$$

Proof. If $i \in \mathscr{M} \backslash \mathscr{N}$, then, by (5), $\left|f_{i}\right|=\left|g_{i}\right| \in \mathscr{D}$. If, moreover, $f_{i}(0)=0$, then $f_{i} \in \mathscr{L}$. If $\varphi(0)=0$, we get similarly $\varphi \in \mathscr{L}$. Thus (8) together with (6) implies (10).

Now let (9) hold. Choose an $s \in \mathscr{N}$ with $f_{s}(0) \neq 0$. Then $g_{s}(0)>0$ and, by (6), $g_{s} \in \mathscr{A}$. Therefore according to (7)

$$
\lim \inf \operatorname{ap} \varphi(t)>0 \quad(t \rightarrow 0+) .
$$

By (5) and 4.6 there are $\alpha_{i} \in \mathscr{A}$ such that $g_{i}=\alpha_{i} \varphi$ for each $i \in \mathscr{M}$. It follows from the relation $g_{s}=\alpha_{s} \varphi$ that $\varphi \in \mathscr{A}$. By (5) and 4.2 we get $\varphi \in \mathscr{L}$. Now let $i \in \mathscr{M} \backslash \mathscr{N}$. Then $f_{i}=g_{i}=\alpha_{i} \varphi$ so that $f_{i} \in \mathscr{A}$. Combining (7) with 4.2 we obtain $f_{i} \in \mathscr{L}$. Thus (9) together with (6) implies (10).

4.10. Proposition. If (11) holds, then there are $\beta_{1}, \ldots, \beta_{m} \in \mathscr{A}$ such that $f_{i}=\beta_{i} \varphi$ for each $i \in \mathscr{M}$.

Proof. By 4.8 (see (5)) and 4.6 we have $\varphi(0)>0$ and there are $\alpha_{i} \in \mathscr{A}$ such that $g_{i}=\alpha_{i} \varphi \quad(i \in \mathscr{M})$. If $i \in \mathscr{M} \backslash \mathscr{N}$, set $\beta_{i}=\alpha_{i}$; if $i \in \mathscr{N}$, define

$$
\begin{cases}\beta_{i}(t)=f_{i}(t) / \varphi(t), & \text { if } \varphi(t)>0, \\ \beta_{i}(t)=\beta_{i}(0) & \text { elsewhere on }[0,1] .\end{cases}
$$

Then $f_{i}=\beta_{i} \varphi$ for each $i \in \mathscr{M}$. It remains to prove that $\beta_{i} \in \mathscr{A}$ for each $i \in \mathscr{N}$. Choose such an $i$. By (6) we have $f_{i} \in \mathscr{A}$. If $f_{i}(0) \neq 0$, then (9) holds so that, according to (10), $\varphi \in \mathscr{A}$ and by (12) we have $\beta_{i} \in \mathscr{A}$. If $f_{i}(0)=0$, then, by (11) and (12), we have $\beta_{i} \in \mathscr{A}$ again.

4.11. Proposition. If $\varphi \in \mathscr{A}$, then (10) holds.

Proof. By (5) and 4.2 we have $\varphi \in \mathscr{L}$. If $\varphi(0)=0$, we apply 4.9. If $i \in \mathscr{N}$, then by (6) we have $f_{i} \in \mathscr{L}$. Thus let $\varphi(0)>0$ and $i \in \mathscr{M} \backslash \mathscr{N}$. By 4.10 we have $f_{i} \in \mathscr{A}$ and the relation $f_{i} \in \mathscr{L}$ follows from (7) and 4.2.

4.12. Lemma. Let $r$ be a natural number, $\delta_{1}, \ldots, \delta_{r} \in(0,1), \delta_{1}+\cdots+\delta_{r} \leqq 1$, $h_{1}, \ldots, h_{r} \in\left(\mathscr{D}^{*}\right)^{+}$. Then $h_{1}^{\delta_{1}} \cdots h_{r}^{\delta_{r}} \in \mathscr{D}^{*}$.

Proof. We may suppose that $\delta_{1}+\cdots+\delta_{r}=1$. Let $t \in(0,1)$. By Hölder's inequality we have $t^{-1} \int_{0}^{t} h_{1}^{\delta_{1}} \cdots h_{r}^{\delta_{r}} \leqq \prod_{j=1}^{r}\left(t^{-1} \int_{0}^{t} h_{j}\right)^{\delta_{j}}$ for each $t \in(0,1)$ which easily implies our assertion. 


\section{DERIVATIVES AND APPROXIMATE CONTINUITY}

In this section we shall apply Propositions 4.8-4.11 and Lemma 4.12 using the norm $|x|=\|x\|_{p} \quad(p>1)$. We shall see that the behavior of derivatives $f_{1}, \ldots, f_{m}$ depends on some properties of the sum of powers of their absolute values. In 5.10 and 5.11 we obtain some results pointing in the opposite direction.

5.1. Notation. For each $p \in(1, \infty)$ and each $x \in R^{m}$ define $\|x\|_{p}$ by $(0)$ and set $Z_{p}=\left\{x \in R^{m} ;\|x\|_{p}=1\right\}$.

5.2. Lemma. Let $p, q \in(1, \infty), p^{-1}+q^{-1}=1, x, y \in R^{m}$. Then $x \cdot y \leqq$ $\|x\|_{p}\|y\|_{q}$; equality holds if and only if

$$
y_{i}=\left|x_{i}\right|^{p-1} \operatorname{sgn} x_{i} \text { for } i=1, \ldots, m \text {. }
$$

Proof. It is well known that $x \cdot y \leqq\|x\|_{p}\|y\|_{q}$ and that equality holds if and only if $x_{i} y_{i} \geqq 0$ and $\left|x_{i}\right|^{p}=\left|y_{i}\right|^{q}$ for each $i$. Since $p / q=p-1$, this equality means the same as $\left|y_{i}\right|=\left|x_{i}\right|^{p-1}$. This easily implies our assertion.

5.3. Proposition. Let $p \in(1, \infty)$. For each $v \in R^{m}$ set $|v|=\|v\|_{p}$. Let $x \in Z_{p}$ and $b=\left(b_{1}, \ldots, b_{m}\right)$, where $b_{i}=\left|x_{i}\right|^{p-1} \cdot \operatorname{sgn} x_{i}$. Then conditions $\left(\mathrm{C}_{1}\right)$ and $\left(\mathrm{C}_{2}\right)$ in 3.4 are fulfilled.

Proof. The validity of $\left(\mathrm{C}_{1}\right)$ is obvious. Define $q$ by $p^{-1}+q^{-1}=1$. We have $x \cdot b=1$ and $\|b\|_{q}=1$. If $y \in Z_{p}$, then $y \cdot b \leqq\|y\|_{p}\|b\|_{q}=1$; if, moreover, $y \cdot b=1$, then we obtain from 5.2 the equalities $b_{i}=\left|y_{i}\right|^{p-1} \operatorname{sgn} y_{i}$ so that $y=x$.

Now let $c=\left(c_{1}, \ldots, c_{m}\right) \in R^{m}, x \cdot c=1$ and $y \cdot c \leqq 1$ for each $y \in Z_{p}$. Set $v=\left(v_{1}, \ldots, v_{m}\right)$, where $v_{i}=\left|c_{i}\right|^{q-1} \operatorname{sgn} c_{i}$. Since $v /\|v\|_{p} \in Z_{p}$, we have $v \cdot c \leqq\|v\|_{p}$ while by 5.2, $v \cdot c=\|v\|_{p}\|c\|_{q}$. It follows that $\|c\|_{q} \leqq 1$. Since $x \cdot c=1 \geqq\|x\|_{p}\|c\|_{q}$, by (13) we have $c_{i}=b_{i}$ so that $c=b$. This proves $\left(\mathrm{C}_{2}\right)$.

5.4. Notation. Let $D$ be the system of all finite derivatives on $R$. Thus, the relation $f \in D$ means that there is a function $F$ differentiable on $R$ such that $F^{\prime}(t)=f(t)$ for each $t \in R$. If, moreover, $f$ is locally integrable, then $\int_{a}^{b} f=F(b)-F(a)$ for all $a, b \in R$.

Let $A$ be the system of all approximately continuous functions on $R$ and let $L$ be the system of all Lebesgue functions on $R$ (i.e., functions $f$ such that $h^{-1} \int_{t}^{t+h}|f-f(t)| \rightarrow 0 \quad(h \rightarrow 0)$ for each $\left.t \in R\right)$. The symbols $b \mathscr{T}$ and $\mathscr{T}^{+}$ will have the same meaning as in 4.1 .

It is well known that $b A \subset L \subset D \cap A$. We shall often apply the fact that nonnegative derivatives are locally integrable. For each $p \in(1, \infty)$ let $Q_{p}$ be the set of all functions $\Phi$ with the following property: There is a natural number $r$, positive numbers $q_{j}$, and functions $h_{j} \in D^{+}(j=1, \ldots, r)$ such that $q_{1}+\cdots+q_{r} \leqq p$ and $\Phi=h_{1}^{q_{1}} \cdots h_{r}^{q_{r}}$. (We see that products of $p$ nonnegative derivatives are in $Q_{p}$, if $p$ is an integer.) 
5.5. Theorem. Let $p \in(1, \infty)$. Let $f_{i} \in D, p_{i} \in(p, \infty)(i=1, \ldots, m)$ and $\sum_{i=1}^{m}\left|f_{i}\right|^{p_{i}} \in Q_{p}$. Then $f_{i} \in L$ for each $i$.

Proof. Set $\Phi=\sum_{i=1}^{m}\left|f_{i}\right|^{p_{i}}$. Let $q_{1}, \ldots, q_{r}$ be as in the definition of $Q_{p}$. Now we apply 4.12 with $\delta_{j}=q_{j} / p$ and 4.8 with $|x|=\|x\|_{p}, \mathscr{N}=\{1, \ldots, m\}$ and $\gamma_{i}(t)=|t|^{p_{i} / p}$.

5.6. Theorem. Let $p \in(1, \infty), f_{1}, \ldots, f_{m} \in D$; set $\varphi=\left(\sum_{i=1}^{m}\left|f_{i}\right|^{p}\right)^{1 / p}$. Then $\varphi \in L$ if and only if $f_{i} \in L$ for each $i$.

Proof. If $\varphi \in L$, we apply 4.11 with $|x|=\|x\|_{p}$ and empty $\mathscr{N}$. The proof that $\varphi \in L$, if $f_{1}, \ldots, f_{m} \in L$, is left to the reader.

Remark 1 . Choosing a nonempty $\mathscr{N} \neq \mathscr{M}$ we may obtain from 4.8-4.12 various other theorems analogous to 5.5 and 5.6. For example, choosing $|x|=\|x\|$, $m=p=2, \mathcal{N}=\{1\}, f_{1}=1, f_{2}=f, \gamma(t)=t^{2}$ we deduce easily from 4.9 that a derivative $f$ is a Lebesgue function if and only if $\sqrt{1+f^{2}}$ is a derivative.

Remark 2. If the functions $f_{1}, \ldots, f_{m}$ and $\varphi=\left(\sum_{i=1}^{m}\left|f_{i}\right|^{p}\right)^{1 / p}$ are derivatives and if $\varphi$ is "not too small," then by 4.10 , the functions $f_{i} / \varphi$ are approximately continuous. Now it is natural to ask whether the relations, say, $1<f_{i} \in D$ and $f_{i} / \varphi \in A \quad(i=1, \ldots, m)$ imply that $\varphi \in D$. Example 3 in 5.14 shows that this is not true even in the simple case $m=p=2$. However, according to Proposition 5.10, we get the desired result $\varphi \in D$, if we replace the requirement $f_{i} \in D$ by $f_{i} \in M$, where $M$ is defined next.

5.7. Definition. Let $M$ be the system of all functions $f \in D$ such that $f g \in D$ for each $g \in b A$.

Remark. It is easy to prove that $L \cup b D \subset M$. A characterization of $M$ will be given in another paper. Here we prove only two simple propositions to give the reader an idea about the "size" of $M$.

5.8. Proposition. We have $A \cap M=L$.

Proof. Let $f \in A \cap M$. Set $f_{0}=(f \vee(-1)) \wedge 1, g=f f_{0}$. Then $g(t)=(f(t))^{2}$ if $|f(t)|<1$, and $g(t)=|f(t)|$ elsewhere on $R$. Hence $|f|<1+g$. Since $f_{0} \in b A$, we have $g \in A \cap D$. By 4.2 we have $f \in L$. The proof of the inclusion $L \subset A \cap M$ is left to the reader.

5.9. Proposition. Let $f \in D$. Suppose that for each $t \in R$ there is a $p \in(1, \infty)$ such that

$$
\underset{h \rightarrow 0}{\limsup } h^{-1} \int_{t}^{t+h}|f|^{p}<\infty
$$

Then $f \in M$.

Proof. Let $g \in b A$ and let $t, p$ be as above. Define $q$ by $p^{-1}+q^{-1}=1$ and set $f_{1}=f-f(t), g_{1}=g-g(t)$. Then $f g=f_{1} g_{1}+v$, where $v \in D$. By 
Hölder's inequality we have

$$
\frac{1}{h} \int_{h}^{t+h}\left|f_{1} g_{1}\right| \leqq\left(\frac{1}{h} \int_{t}^{t+h}\left|f_{1}\right|^{p}\right)^{1 / p} \cdot\left(\frac{1}{h} \int_{t}^{t+h}\left|g_{1}\right|^{q}\right)^{1 / q} \quad \text { for each } h \neq 0 \text {. }
$$

It is easy to see that in (14) we may replace $f$ by $f_{1}$. Since $\left|g_{1}\right|^{q} \in b A \subset D$, the second factor in $(15)$ tends to $0(h \rightarrow 0)$. This shows that $f g \in D$.

5.10. Proposition. Let $|\cdot|$ be a norm on $R^{m}$. Let $f_{1}, \ldots, f_{m} \in M, \alpha_{1}, \ldots$, $\alpha_{m} \in A$. Set $f=\left(f_{1}, \ldots, f_{m}\right), \alpha=\left(\alpha_{1}, \ldots, \alpha_{m}\right), \varphi=|f|$ and suppose that $f=\alpha \varphi$. Then $\varphi \in M$.

Proof. Obviously $\varphi=|\alpha| \varphi$ so that $|\alpha|=1$ on the set $S=\{t \in R ; \varphi(t)>0\}$. Defining $\gamma=k^{2} \vee(\alpha \cdot \alpha)$ (see (1)) we have $\gamma \in A, \gamma=\alpha \cdot \alpha$ on $S, \alpha \cdot f=\gamma \varphi$ on $R$. Let $T=\{t \in R ;\|\alpha(t)\| \leqq k\}$. On $T$ we have $\gamma=k^{2}$ so that $\|\alpha\| \leqq \gamma / k$; on $R \backslash T$ we have $\gamma=\|\alpha\|^{2}$ so that $\|\alpha\|=\gamma /\|\alpha\|<\gamma / k$. We see that $\|\alpha\| / \gamma \leqq k^{-1}$ on $R$ whence $\alpha_{i} / \gamma \in b A, \alpha_{i} f_{i} / \gamma \in M(i=1, \ldots, m), \varphi=\alpha f / \gamma$.

5.11. Theorem. Let $p \in(1, \infty)$ and let $f_{1}, \ldots, f_{m} \in M$. Define $\varphi=$ $\left\|\left(f_{1}, \ldots, f_{m}\right)\right\|_{p}, \Phi=\varphi^{p}$ and suppose that

$$
\liminf \operatorname{ap}_{y \rightarrow t} \varphi(y)>0 \text { for each } t \in R .
$$

Then the following conditions are equivalent:

(i) $\Phi \in Q_{p}$;

(ii) $\varphi \in D$;

(iii) there are $\alpha_{i} \in A$ such that $f_{i}=\alpha_{i} \varphi$ for $i=1, \ldots, m$;

(iv) there are functions $\psi, \alpha_{1}, \ldots, \alpha_{m}$ such that $\psi \in D^{+}, \alpha_{i} \in A$, and $f_{i}=\alpha_{i} \psi$ for $i=1, \ldots, m$;

(v) $\varphi \in M$.

Proof. If (i) holds, we apply 4.12 as in the proof of 5.5 , then 4.8 with $|x|=$ $\|x\|_{p}$ and from (5) we get (ii). If (ii) holds, then (iii) follows from 4.10. If (iii) holds, then by 5.10 , we have $\varphi \in D$ so that we may choose $\psi=\varphi$ in (iv). Now suppose that (iv) holds. Set $f=\left(f_{1}, \ldots, f_{m}\right), \alpha=\left(\alpha_{1}, \ldots, \alpha_{m}\right)$. It is easy to see that $\varphi=\|f\|_{p}=\psi\|\alpha\|_{p}$ and that $\|\alpha\|_{p} \in A$. If $t \in R$ and $\|\alpha(t)\|_{p}=0$, then (16) implies $\operatorname{limap} \operatorname{ap}_{y \rightarrow t} \psi(y)=\infty$ which is impossible because $\psi \in D^{+}$. Hence $\alpha \cdot \alpha>0$ on $R$. Set $\beta=\alpha\|\alpha\|_{p} /(\alpha \cdot \alpha)$. By (1) $\|\beta\|=\|\alpha\|_{p} /\|\alpha\| \leqq k^{-1}$ so that $\beta \cdot f \in M$ and $\beta \cdot f=(\alpha \cdot \alpha \psi)\|\alpha\|_{p} /(\alpha \cdot \alpha)=\psi\|\alpha\|_{p}=\varphi$. This proves (v). If (v) holds, then, obviously, $\Phi=\varphi^{p} \in Q_{p}$ which completes the proof.

Remark. We conclude the paper with three examples. The first shows that the requirement (16) cannot be replaced by " $\varphi>0$ on $R$." The second reminds us that in 4.11 we supposed that $\varphi \in \mathscr{D}^{*}$ (see 4.7); the assumption that $\varphi$ is integrable would not suffice. The third refers to Remark 2 in 5.6.

5.12. Example 1. Let $\gamma$ be a bounded derivative such that $\gamma=1$ on $(-\infty, 0]$, $\gamma$ is continuous and nonnegative on $(0, \infty)$ and that the upper density of the 
set $S=\{t \in R ; \gamma(t)=0\}$ at 0 is positive. Let $\mu$ be a bounded continuous function on $R$ such that $\mu(0)=0$ and $\mu(t)>0$ for $t \neq 0$. Further set $f=3 \gamma+4 \mu, g=4 \gamma+3 \mu, \varphi=\sqrt{f^{2}+g^{2}}$. Then $f, g \in D$ and from the inequalities $5 \gamma \leqq \varphi \leqq 5(\gamma+\mu)$ it follows easily that $\varphi \in D$. It is obvious that all the functions $f, g$, and $\varphi$ are positive; since they are bounded, they belong to $M$. However, $(f / \varphi)(0)=\frac{3}{5}$ and $f / \varphi=\frac{4}{5}$ on $S$. Therefore $f / \varphi \notin A$.

5.13. Example 2. Let $x_{2}>x_{3}>\cdots, x_{n} \rightarrow 0, x_{n+1} / x_{n} \rightarrow 1$. Set $Q=5 / \sqrt{2}$, $d_{n}=x_{n-1}-x_{n}, \quad y_{n}=x_{n}+d_{n} / n, v_{n}=\left(x_{n}+x_{n-1}\right) / 2, w_{n}=v_{n}+d_{n} / n$ $(n=3,4, \ldots)$. Let $f, g$ be functions on $R$ continuous on $(0, \infty)$ such that $f=g=Q$ on $(-\infty, 0], f=3, g=4$ on $\left(y_{n}, v_{n}\right), f=4, g=3$ on $\left(w_{n}, x_{n-1}\right), f \geqq 3, g \geqq 3$ on $R$ and $\int_{x_{n}}^{x_{n-1}} f=\int_{x_{n}}^{x_{n-1}} g=Q d_{n}$. (Such functions exist, because $Q>(3+4) / 2$.) Set $S=\bigcup_{n=3}^{\infty}\left(\left(x_{n}, y_{n}\right) \cup\left(v_{n}, w_{n}\right)\right)$. Since $x_{n+1} / x_{n} \rightarrow 1$, we have $f, g \in D$ and $S$ has density 0 at 0 . It is easy to see that the function $\varphi=\sqrt{f^{2}+g^{2}}$ is locally integrable, $\varphi \in A$ (because $\varphi=5$ on $\left.\left(-\infty, x_{2}\right) \backslash S\right)$ and that $f, g \notin A$.

5.14. Example 3. Let $x_{n}, d_{n}, y_{n}$ be as in 5.13. Set $J_{n}=\left[x_{n}, y_{n}\right], L_{n}=$ $\left[x_{n}, x_{n-1}\right] \quad(n=3,4, \ldots)$. It is easy to construct a function $v$ on $R$ continuous on $(0, \infty)$ such that $v=1$ on $(-\infty, 0], v \geqq \frac{1}{2}$ on $R, v=\frac{n}{2}$ on $J_{n}$ (so that $\int_{J_{n}} v=d_{n} / 2$ ) and $\int_{L_{n}} v=d_{n}$. Let $\mu$ be a nonconstant function continuous on $[0,1]$ such that $\int_{0}^{1} \mu=0, \mu(0)=\mu(1)=0$ and that $-\frac{1}{2} \leqq \mu \leqq 1$. Set $q=\int_{0}^{1} \sqrt{1+\mu^{2}}$ (so that $q>1$ ). Define a function $h$ on $R$ setting $h(t)=\mu\left(\left(t-x_{n}\right) /\left(y_{n}-x_{n}\right)\right)$ for $t \in J_{n} \quad(n=3,4, \ldots)$ and $h(t)=0$ elsewhere on $R$. Now set $f=v \cdot(3+4 h), g=v \cdot(4-3 h), \varphi=\sqrt{f^{2}+g^{2}}$. Then $\varphi=5 v \sqrt{1+h^{2}}$. It is easy to see that $f, g$, and $\varphi$ are continuous on $R \backslash\{0\}$ and that $\int_{J_{n}} h=0, \int_{J_{n}} \sqrt{1+h^{2}}=q\left(y_{n}-x_{n}\right)$. Thus $\int_{J_{n}} v h=0$ and $\int_{J_{n}} v \cdot \sqrt{1+h^{2}}=q d_{n} / 2$. It follows that $\int_{L_{n}} f=3 d_{n}, \int_{L_{n}} g=4 d_{n}$, $\int_{L_{n}} \varphi=5 d_{n}(q+1) / 2$. This combined with $x_{n+1} / x_{n} \rightarrow 1$ yields $f, g \in D$ and $\lim _{t \rightarrow 0+} \frac{1}{t} \int_{0}^{t} \varphi=5(q+1) / 2>5=\varphi(0)$ so that $\varphi \notin D$. On $R \backslash \bigcup_{n=3}^{\infty} J_{n}$ we have $f \geqq \frac{3}{2}, g \geqq 2, f / \varphi=\frac{3}{5}, g / \varphi=\frac{4}{5}$; in particular, $f / \varphi, g / \varphi \in A$. The inequalities $-\frac{1}{2} \leqq h \leqq 1$ imply that $f \geqq \frac{n}{2}, g \geqq \frac{n}{2}$ on $J_{n}$. Thus $f>1$, $g>1$ on $R$.

\section{REFERENCES}

1. Jan Mařík and Clifford E. Weil, Products of powers of nonnegative derivatives, Trans. Amer. Math. Soc. 276 (1983), 361-373.

2. __ Sums of powers of derivatives, Real Anal. Exchange 13 (1987-88), 180-182.

3. A. Bruckner, J. Mařik, and C. Weil, Some aspects of products of derivatives, Amer. Math. Monthly (to appear).

Department of Mathematics, Michigan State University, East Lansing, Michigan 48824 